\title{
Decreased Membrane Mechanical Stability and In Vivo Loss of Surface Area Reflect Spectrin Deficiencies in Hereditary Spherocytosis
}

\author{
Joel Anne Chasis, Peter Agre, and Narla Mohandas \\ Departments of Medicine and Laboratory Medicine, Cancer Research Institute, University of California, \\ San Francisco, California 94143; and the Departments of Medicine and Cell Biology/Anatomy, \\ Johns Hopkins University School of Medicine, Baltimore, Maryland 21205
}

\begin{abstract}
Whereas marked variations in the clinical manifestations of hereditary spherocytosis have long been recognized, we have only recently begun to define the molecular basis for this heterogeneity. An important unanswered question is whether decreased spectrin results in reduced membrane mechanical stability, and if this reduction in membrane mechanical stability can be related to in vivo surface area loss. Using the ektacytometer, we quantitated membrane surface area and stability in erythrocytes from 18 individuals with hereditary spherocytosis and deficiencies of spectrin (30-80\% of normal spectrin level). Membrane mechanical stability was reduced and the magnitude of the reductions correlated with the spectrin content. Moreover, the reductions in mechanical stability correlated with in vivo loss of membrane surface area. These data indicate that decreased spectrin content results in reduced membrane mechanical stability and surface area loss in vivo. We conclude that partial deficiencies of spectrin, reductions in membrane mechanical stability, and loss of membrane surface area are directly related and are major features determining the heterogeneous clinical manifestations of hereditary spherocytosis.
\end{abstract}

\section{Introduction}

It has long been recognized that the inheritance pattern and clinical manifestations of hereditary spherocytosis (HS) ${ }^{1}$ are heterogeneous $(1,2)$. The phenotype of spherocytic red blood cells can be inherited in an autosomal dominant or nondominant manner, and the clinical course can range from severe hemolytic anemia to a mild, compensated hemolytic state. Recent studies have begun to delineate the molecular basis for this heterogeneity. A moderate decrease in spectrin was noted in erythrocytes from individuals with the dominantly inherited form of the disorder, whereas more marked spectrin deficiencies were seen in a group of families with nondominantly inherited disease $(3,4)$. Individuals from seven of these eight

Address reprint requests to Dr. Joel Anne Chasis, Cancer Research Institute, M-1282/Box 0128, University of California, San Francisco, CA 94143.

Received for publication 22 September 1987 and in revised form 14 March 1988.

1. Abbreviations used in this paper: DI, deformability index; HE, hereditary elliptocytosis; HPP, hereditary pyropoikilocytosis; HS, hereditary spherocytosis.

J. Clin. Invest.

(c) The American Society for Clinical Investigation, Inc.

0021-9738/88/08/0617/07 \$2.00

Volume 82, August 1988, 617-623 nondominant families were found to be homozygotes for a variation in the spectrin $\alpha$ II domain tryptic peptides (5). In all of these spectrin-deficient individuals, the increase in osmotic fragility and rate of hemolysis appear to relate to the membrane spectrin content (4). Recently, electron microscopic studies indicate that in spectrin-deficient HS red cells, the membrane skeleton is disordered in proportion to the degree of spectrin deficiency (6).

Several other subgroups of HS have now been reported in which a second skeletal protein defect exists in addition to spectrin deficiency. Two families with dominantly inherited HS have been described in which the affected members are apparently heterozygous for a spectrin variant with abnormal protein 4.1 binding characteristics $(7,8)$ resulting from oxidative changes in the $\beta$ subunit of spectrin (9). Spherocytosis with ankyrin deficiency and 4.2 deficiency have also been identified $(10,11)$.

To establish that in HS, deficiencies of membrane spectrin are directly responsible for generation of spherocytes with reduced surface area, it is necessary to document two important functional relationships: first, that decreased spectrin results in reduced membrane mechanical stability; and second, that loss of surface area in vivo reflects reduced membrane mechanical stability. If such quantitative relationships between surface area, mechanical stability, and membrane spectrin content can be documented, it is possible to conclude that spectrin deficiency is a major factor contributing to the pathophysiology of HS.

To address these questions, the membrane surface area and mechanical stability were characterized in a group of patients with $\mathrm{HS}$ and quantitative deficiencies of spectrin, determined by RIA to range from 30 to $80 \%$ of the normal spectrin level. Membrane surface area and membrane mechanical stability were quantitated using the ektacytometer. In the 18 individuals studied, we found that the membrane mechanical stability was reduced and that the extent of this reduction correlated with the decrease in spectrin content. Moreover, the reduction in mechanical stability was directly related to the extent of membrane surface area loss in vivo.

\section{Methods}

Reagents. Dextran was purchased from Pharmacia Fine Chemicals, Uppsala, Sweden; polyvinylpyrrolidone from Sigma Chemical Co., St. Louis, MO; and Stractan II from St. Regis Paper Co., New York, NY.

Blood was drawn by venipuncture, anticoagulated with acid citrate dextrose, packed in ice, and analyzed within $24 \mathrm{~h}$ of phlebotomy. In all cases, a sample was drawn from a normal control simultaneously and processed identically to the patient samples. All of the patients involved in this study had been splenectomized.

Quantitation of spectrin and band 3. Erythrocyte spectrin and band 3 contents were determined by RIA as previously described (3). Briefly, 
spectrin in a known number of detergent-solubilized erythrocytes was used to compete with ${ }^{125} \mathrm{I}$-labeled spectrin for a limiting number of sites on immunoadsorbents prepared from antispectrin IgG adsorbed onto formalin-fixed, protein A-bearing Staphylococci. In all cases, the spectrin content of the patient sample is expressed as the percent of the normal control. An RIA using ${ }^{125}$ I-labeled $43,000-M_{\mathrm{r}}$ cytoplasmic fragment of band 3 was performed similarly.

The density of spectrin on the membrane was determined as described (3) by calculating the ratio of spectrin to the anion transporter (band 3). Erythrocyte membranes were prepared by lysis of washed erythrocytes in $7.5 \mathrm{mM} \mathrm{NaPO}_{4}(\mathrm{pH} 7.5)$. The washed membranes were dissolved in SDS and electrophoresed through 3.5-17\% polyacrylamide slab gels using the Fairbanks buffer system (12). The slabs were stained with Coomassie Brilliant Blue R250 and destained until the background was colorless. The bands corresponding to spectrin and band 3 were excised, the dye was eluted in $25 \%$ (vol/vol) pyridine in water, and the absorbance of the spectrin eluate and the band 3 eluate were measured at $605 \mathrm{~nm}$ (13). The ratio of the absorbances (spectrin/ band 3 ratio) was generally $\sim 1.0$ for normals, and the ratios for all patients were normalized with those obtained for the controls. This was done by determining spectrin/band 3 ratios from normal membranes prepared at the same time the patient samples were prepared. The patient and control membranes were then electrophoresed in alternate lanes of the same gels and each patient was normalized to a normal control.

The spectrin content and spectrin/band 3 values used in this study are taken from an earlier study (3). The use of this previously obtained biochemical data is appropriate for this study because spectrin content by RIA and spectrin/band 3 ratios do not change over time in splenectomized individuals (3).

Membrane mechanical stability and deformability measurements. Resealed membranes for stability and deformability measurements were prepared by a procedure adopted from Johnson (14). The erythrocytes were washed three times in $5 \mathrm{mM}$ Tris and $140 \mathrm{mM} \mathrm{NaCl}(\mathrm{pH}$ 7.4), and then lysed in $40 \mathrm{vol}$ of $7 \mathrm{mM} \mathrm{NaCl}$ and $5 \mathrm{mM}$ Tris (pH 7.4). The membranes were then pelleted by centrifugation, resuspended in $10 \mathrm{vol}$ of $5 \mathrm{mM}$ Tris and $140 \mathrm{mM} \mathrm{NaCl}(\mathrm{pH} \mathrm{7.4)}$, and incubated 30 min at $37^{\circ} \mathrm{C}$ for resealing.

For deformability measurements, resealed membranes, prepared as described above, were suspended in $3 \mathrm{ml}$ Stractan II (290 mosmol, 22 centipoise [cp], $\mathrm{pH} 7.4$ ) and exposed to an increasing shear stress $\left(0-125 \mathrm{dyn} / \mathrm{cm}^{2}\right)$ in the ektacytometer. The change in their laser diffraction pattern from circle to ellipse was measured photometrically, producing a signal, designated deformability index (DI), which quantitates cell ellipticity. By an automatic image analysis system, the DI is recorded as a continuous function of applied shear stress. For resealed membranes, the shear stress required to obtain a defined value of DI is determined by the property of membrane deformability without contributions from either internal viscosity or cell geometry (15). There is a correlation between changes in deformability measured by this technique and those measured using the micropipette $(16,17)$. Analysis of the DI curve generated by the ektacytometer thus provides a measure of membrane deformability (18).

For mechanical stability measurements, the resealed membranes were pelleted by centrifugation and $100 \mu \mathrm{l}$ of $40 \%$ membrane suspension was mixed with $3 \mathrm{ml}$ dextran $(40,000 \mathrm{~mol} \mathrm{wt}, 35 \mathrm{~g} / 100 \mathrm{ml}$ in 10 $\mathrm{mM}$ phosphate buffer, $\mathrm{pH} 7.4$, viscosity $95 \mathrm{cp}$ ) and subjected continuously to $750 \mathrm{dyn} / \mathrm{cm}^{2}$ in the ektacytometer $(19,20)$. Under this stress, the membranes progressively fragment, generating undeformable spheres. This process is detected as a time-dependent decrease in the DI. The time required for the DI to fall to $60 \%$ of its maximum value is termed $t_{60}$ and is taken as a measure of membrane stability. An analysis of the shear-induced fragments after exposure of resealed membranes to the shear stress of the mechanical stability assay showed that the membrane protein composition of the fragments was the same as that of both unsheared resealed membranes and of residual membranes after fragmentation (20). The protein/lipid ratios were also very similar for these three preparations, and the ratios of protein 4.1 , spectrin, and actin to band 3 were within $7 \%$ of each other (20). The shear-induced fragmentation thus results in the disruption of protein-protein interactions in the membrane skeleton, generating spherical vesicles with the same protein composition as that of the intact membranes from which they were derived.

Measurement of the mean membrane surface area. The surface area of red blood cells was measured using osmotic gradient ektacytometry, an assay in which whole cell deformability is measured as a continuous function of suspending medium osmolality (21). For these measurements, we prepared gradients from two solutions of $4 \%$ polyvinylpyrrolidone and phosphate-buffered $\mathrm{NaCl}$, one adjusted to 50 and the other to $900 \mathrm{mosmol} / \mathrm{kg}$. The gradients were mixed in the first stage of a three-stage mixing chamber of a gradient former (Beckman Instrument Co., Fullerton, CA). Packed red cells (70-80\% hematocrit) were pumped into the second stage of the chamber by an infusion pump (model No. 906; Harvard Apparatus Co., Inc., The Ealing Corp., South Natick, MA) and mixed with the gradient to a final hematocrit of $0.2 \%$. Thorough mixing was ensured by passage of the cell suspension through the third stage of the mixing chamber. The suspension was then pumped through a conductivity meter (Wescan Instruments, Inc., Santa Clara, CA) to continuously monitor its conductivity, and finally into the ektacytometer for measurement of cellular deformability, at a constant shear stress of $170 \mathrm{dyn} / \mathrm{cm}^{2}$. The osmolality at which the deformability index reaches a minimum in the hypotonic region of the gradient has been shown to be the same as the osmolality at which $50 \%$ of the cells will hemolyze in a standard osmotic fragility test (21). This point thus provides a measure of the average surface area/volume ratio of the population of cells studied. The cells attain their maximally deformed state at the physiologically relevant osmolality of 290 mosmol. In the presence of normal membrane deformability, this maximum value of deformability index has previously been shown to be quantitatively related to the membrane surface area (21).

\section{Results}

Membrane surface area. To evaluate the surface area of spectrin-deficient erythrocytes, the cells were examined by osmotic gradient ektacytometry. In this assay the DI is measured as a continuous function of the osmolality of the suspending medium. The normal range and three representative curves of cells with 82,59 , and $31 \%$ of the normal spectrin content (determined by RIA) are shown in Fig. 1. A right shift of the minimum point in the hypotonic region is seen in the spectrin-deficient cells and is consistent with a decrease in cell surface/volume ratio and increased osmotic fragility observed in spherocytosis. A decrease in the maximum DI is also present in the spectrin-deficient cells, implying reduced membrane surface area. It can be seen that the greater the spectrin deficiency, the greater the loss of surface area. This reduction in surface area is, in fact, responsible for the decreased surface area/volume ratio.

Membrane deformability. To ensure that the decrease in the maximum DI observed in the spectrin-deficient cells was due to surface area loss and not decreased membrane deformability, we measured the deformability of these membranes using the ektacytometer. The deformability curves of membranes prepared from the cells with 82,59 , and $31 \%$ of the normal spectrin content are shown in Fig. 2 . It can be seen in the curves for the membranes with 82 and $59 \%$ spectrin content, that after the initial rapid rise in DI, very little further increase in DI was observed with application of increased shear stress. If membranes with decreased deformability were being examined, the DI would increase with increasing applied 


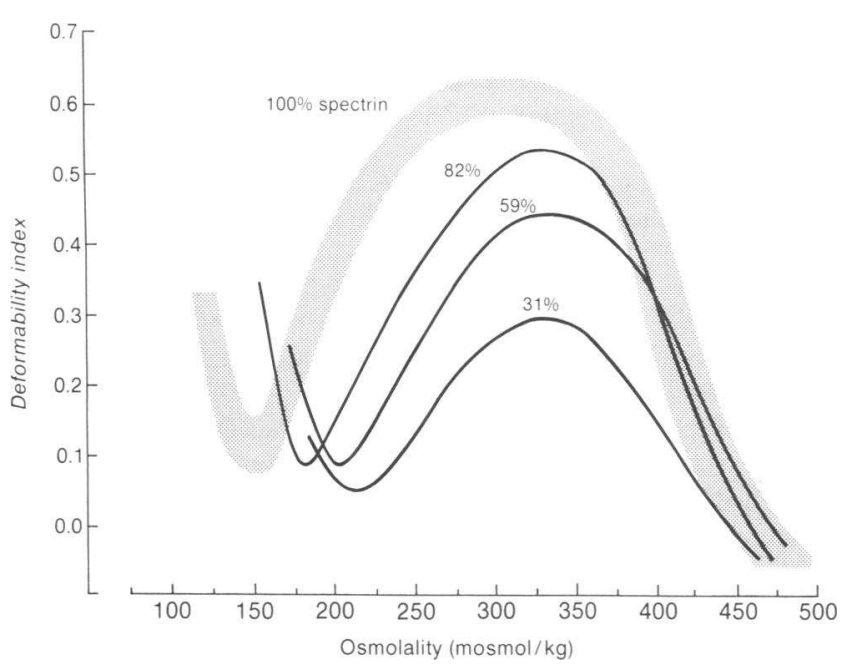

Figure 1. Osmotic gradient ektacytometry of red cells with varying degrees of spectrin deficiency. The minimum DI observed in the hypotonic region is shifted to the right of control (shaded area) in the spectrin-deficient cells (-), with the most severely deficient cells shifted furthest to the right. This indicates a decrease in cell surface/ volume ratio in the spectrin-deficient samples. The maximally obtainable DI of the spectrin-deficient cells is decreased, implying reduced membrane surface area. The more pronounced the spectrin deficiency, the greater the loss of surface area. Spectrin content of these cells was determined by RIA.

shear stress, and with a sufficiently large value of applied shear stress, the membranes would attain a maximum DI comparable to that obtained in normal membranes (22). However, the inability of these HS membranes to reach a normal maximum DI value is consistent with loss of surface area, and not with decreased membrane deformability. Recent micropipette studies also confirm that these spectrin-deficient HS erythrocytes have normal or increased membrane deformability (decreased shear modulus values) (23). Because of extensive loss of surface area, it is difficult to deduce information regarding membrane deformability for the severely spectrin-deficient

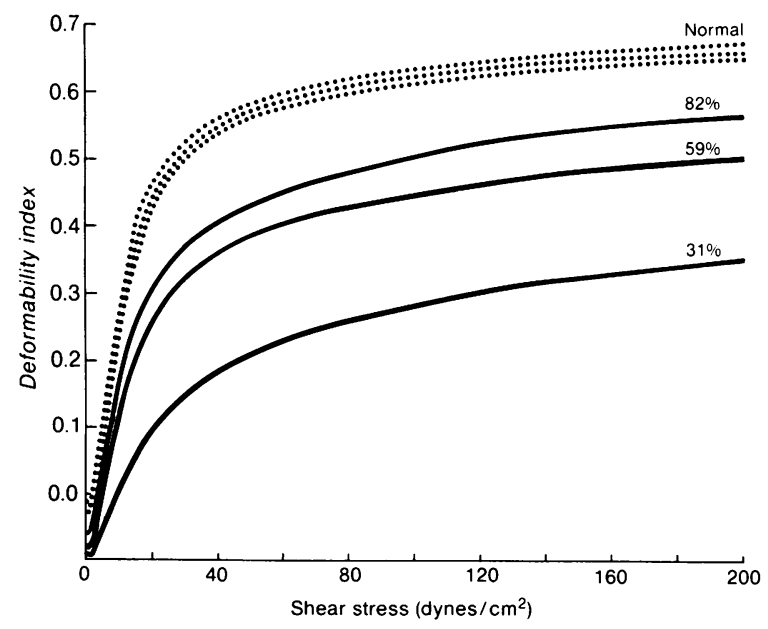

Figure 2. Deformability of membranes with varying degrees of spectrin deficiency. The DI of the spectrin-deficient membranes (-), after an initial rapid rise, plateaus despite continued application of shear stress. This result indicates normal membrane deformability. cells with $31 \%$ spectrin content. Together, these findings imply that the decrease in the maximum DI observed during osmotic gradient ektacytometry was indeed a result of surface area loss.

Relationship between spectrin level and membrane surface area. Spectrin levels were found to reflect membrane surface area with strikingly high significance (Fig. 3). This is consistent with a correlation between the erythrocyte spectrin content and increased osmotic fragility (4). Osmotic gradient ektacytometry provided us with an opportunity to directly assess mean surface area of the cells without confounding influences of cell volume changes. This contrasts with osmotic fragility measurements, in which it is not possible to separate the individual contributions of cell surface area and cell volume to the observed changes (21). We related surface area to membrane spectrin measured by two different approaches, the measurement of spectrin content determined by spectrin RIA (Fig. 3 $A$ ), and the measurement of the relative density of spectrin on the membrane determined by spectrin/band 3 ratio (Fig. $3 B$ ). The relationships of surface area with spectrin content and spectrin density are best fit with curvilinear equations. The correlation of surface area with spectrin density appears more linear, reflecting the partial compensation for very low spectrin content by secondary loss of unsupported membrane. This increases the relative spectrin density on the membrane, expressed as the spectrin/band 3 ratio, since the denominator is also reduced. These results allow us to suggest that spectrin density is a better reflection of surface area than spectrin content. The data for the patients with the dominant form of HS fall on the curves established for the nondominant patients.

To examine whether the spectrin/band 3 ratio is an adequate measure of spectrin density, we studied the correlation between band 3 and surface area. As shown in Fig. 4, there is a tight correlation between the absolute amount of band 3 , as measured by RIA, and the surface area, as measured by ektacytometry. From these data, we conclude that band 3 is not selectively lost from the membrane during in vivo membrane fragmentation and that spectrin/band 3 ratio is a reasonable measure of the density of spectrin assembled on the membrane.

Membrane stability and its relationship to spectrin content. The membrane mechanical stability of the samples was quantitated by monitoring the decrease in DI, at constant applied shear stress, as a function of time (Fig. 5). Since the DI is a measure of shear-induced membrane ellipticity, it decreases as a function of time as the membrane fragments into nondeformable spheres. The fragmentation pattern of normal membranes is shown in the top shaded curve. The DI of the spectrin-deficient membranes decreased more rapidly with time when compared with membranes of normal spectrin content. The extent of this reduction in mechanical stability was also related to the spectrin content of the membrane.

The relationship between membrane mechanical stability and spectrin content of erythrocytes from all the patients was evaluated, and these parameters are related by a linear equation with striking significance (Fig. 6). Interestingly, the projection crosses the abscissa (mechanical stability $=0$ ) at spectrin content of $\sim 25 \%$ of normal. Six individuals have been identified with $30-40 \%$ of the normal spectrin content, and all experienced severe hemolysis requiring regular transfusions and splenectomy as infants (4); however, no patient has yet been identified with $<30 \%$ of the normal spectrin content, and deficiencies of this magnitude may be lethal. 


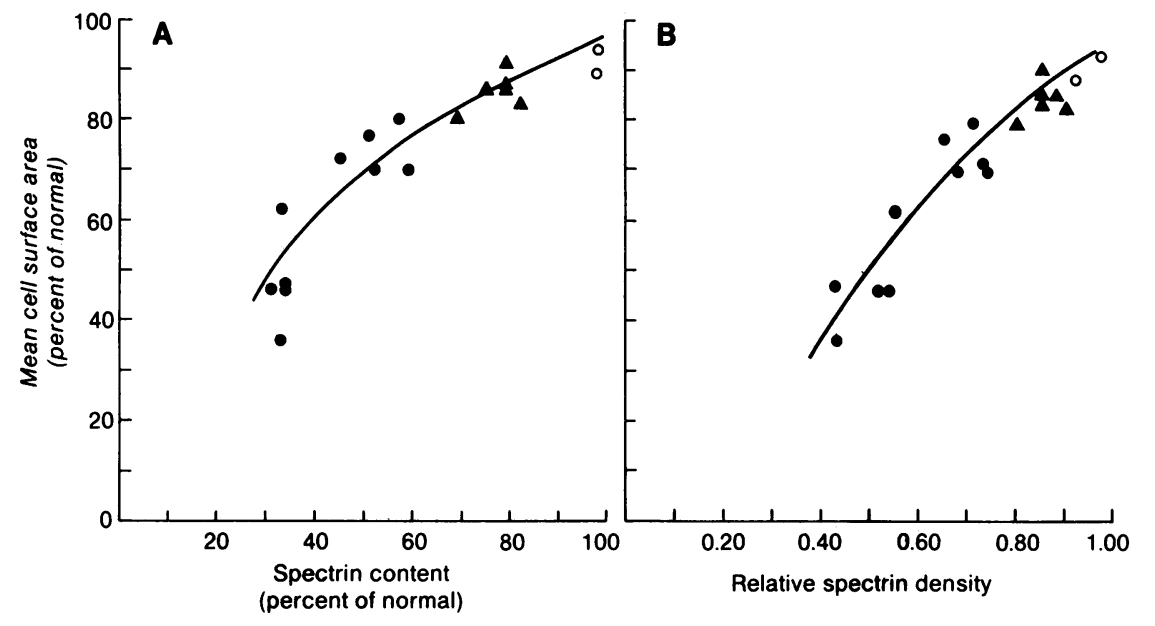

Figure 3. Mean cell surface area and membrane spectrin levels. $(A)$ Spectrin content measured by RIA. The decrease in cell surface area is proportional to the membrane spectrin content in nondominant HS homozygotes (•) and heterozygotes (O). The data for the dominant HS patients $(\Delta)$ fall on the curves established for the nondominant patients. The mean surface area $(S A)$ is related to spectrin content $(S C)$ by the equation $S A=0.96$ $+0.41 \ln (S C) .(r=0.927$. $)(B)$ Mean cell surface area and membrane spectrin density as measured by spectrin/band 3 ratios. The loss of cell surface area is directly related to the decrease in membrane spectrin density expressed as a spectrin/band 3 ratio in homozygous ( $\bullet$ ) and heterozygous (0) nondominant HS. The values for dominant HS ( $\Delta$ ) fall on the curves established for the nondominant form. The mean surface area $(S A)$ is related to the spectrin density $(S D)$ by the equation $S A=0.95$ $+0.65 \ln (S D) .(r=0.954$. $)$
The measurements of membrane stability and spectrin density best fit a curvilinear equation (Fig. 7), whereas the data for membrane stability and spectrin content are best fit by a linear equation (Fig. 6). Membrane stability thus more accurately reflects spectrin content than spectrin density. This is in contrast to our observations regarding surface area, from which it appears that spectrin density more accurately reflects surface area.

Relationship between mechanical stability and membrane surface area. The relationship between membrane surface area and mechanical stability in spectrin-deficient spherocytosis is shown in Fig. 8. As would be expected from the observed relationships between spectrin content, surface area, and mechanical stability, the reduction in mechanical stability was related to the loss of membrane surface area. This observed relationship between these two measured parameters implies that reduced membrane mechanical stability can account for in vivo fragmentation and consequent loss of surface area.

Relationship between membrane mechanical stability and spectrin content in other membrane abnormalities. We also examined whether the same relationship between membrane stability and spectrin density that exists in spectrin-deficient HS erythrocytes also applies to erythrocytes with other molecular defects involving spectrin (Fig. 7). To do this, we replotted data using the previously reported figures for spectrin levels (24-27). The degree of membrane stability of erythrocytes from an individual with dominant HS, due to an abnormal spectrin/protein 4.1 association and a secondary reduction in membrane spectrin density (spectrin/band 3 ratio $=0.80)(24)$, could in large part be accounted for by reduction in spectrin density. In contrast, membranes from three individuals with hereditary elliptocytosis (HE) due to an elongated $\alpha$ spec-

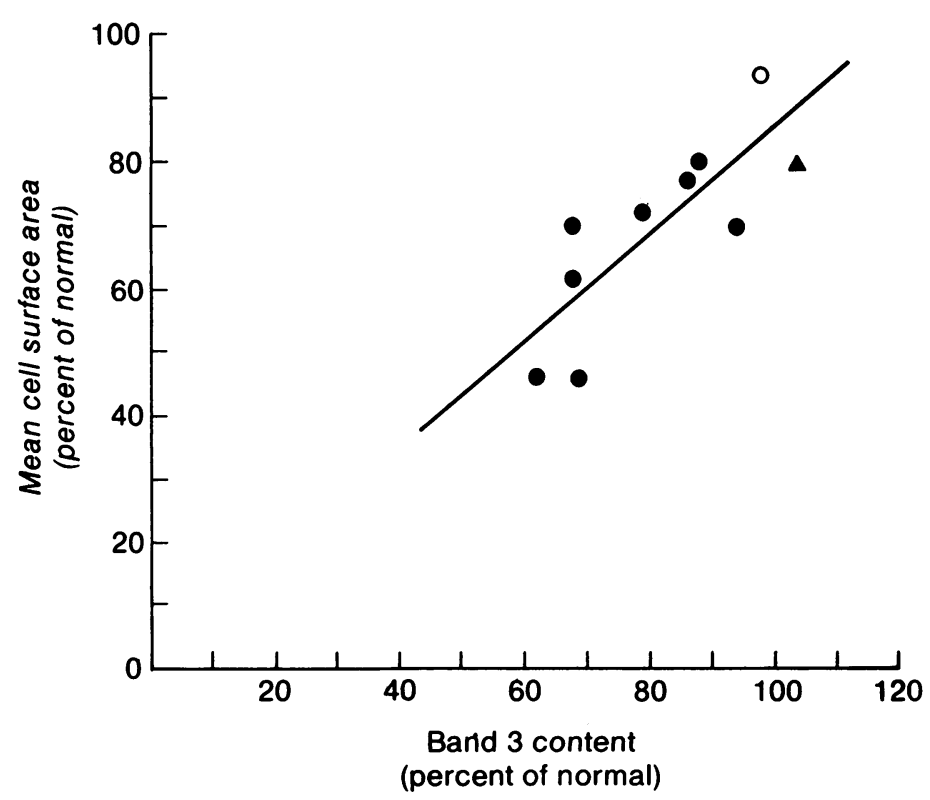

Figure 4. Mean cell surface area and membrane band 3 content as measured by RIA. The mean surface area $(S A)$ is linearly related to band 3 content $(B 3)$ by the equation $S A=2.03+0.83$ $B 3$. $(r=0.806$. $) \bullet$, homozygous nondominant HS; $O$, heterozygous non-dominant HS; $₫$, dominant HS. 


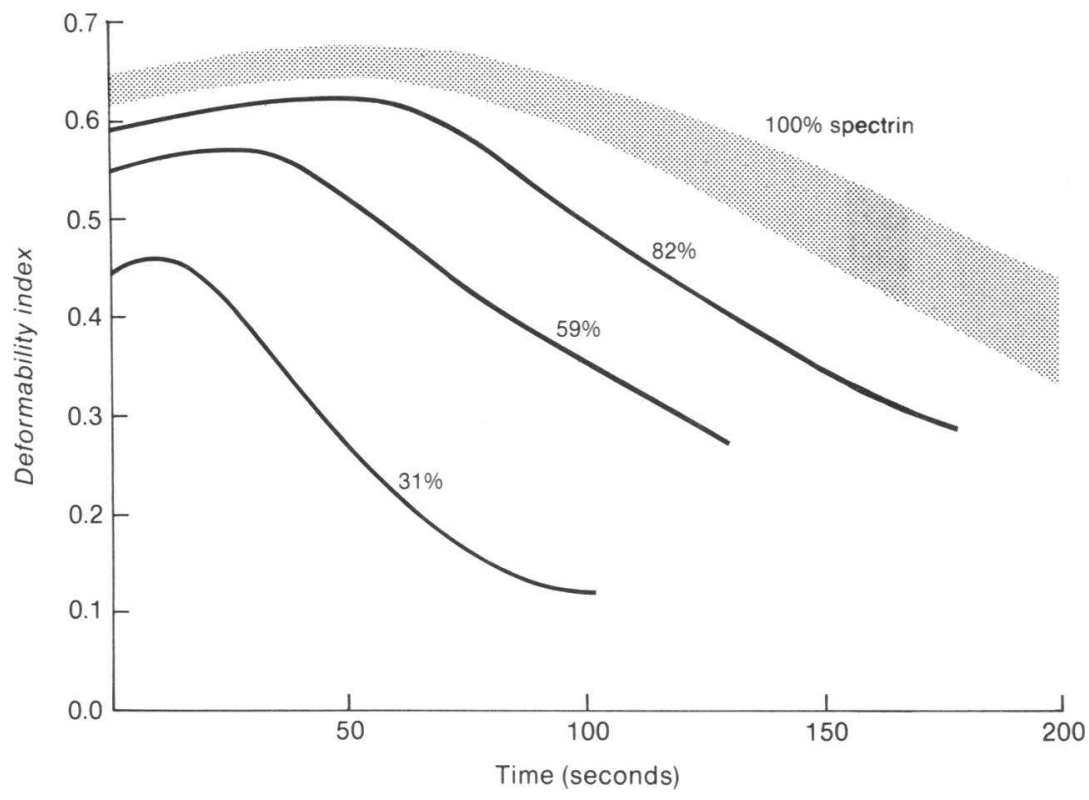

Figure 5. Stability of membranes with varying degrees of spectrin deficiency. The DI of erythrocyte membranes deficient in spectrin $(-)$ decreased more rapidly with time than normal erythrocyte membranes (shaded area) when exposed to constant shear stress, implying decreased mechanical stability. trin mutant, and small secondary reductions in spectrin density (spectrin/band 3 ratio $=0.91-0.99$ ) have markedly reduced stability compared with spectrin-deficient HS membranes "with comparable spectrin content (25). Similarly, discordance between stability and spectrin content was observed in three other individuals with $\mathrm{HE}$ and $\alpha$ spectrin mutants resulting in abnormalities in the alpha I domain of spectrin and spectrin densities of 0.95-1.0 (26). Finally, a patient

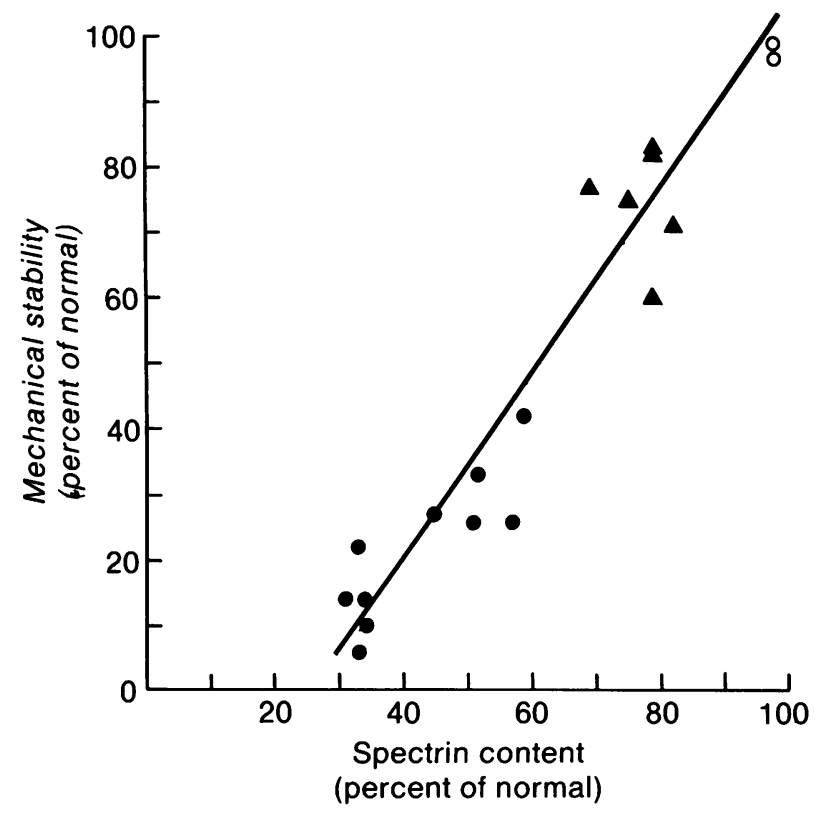

Figure 6. Membrane spectrin content and mechanical stability. The decrease in membrane mechanical stability is proportional to the membrane spectrin content as measured by RIA for individuals homozygous ( $\bullet$ ) or heterozygous ( $(0)$ for nondominant HS. The data for individuals with dominant HS ( $\Delta$ ) fall on the curves established for the nondominants. The relationship between spectrin content $(S C)$ and mechanical stability $(M S)$ is related linearly as $M S=-0.34$ $+1.36 S C(r=0.964)$. with hereditary pyropoikilocytosis (HPP) and membrane spectrin density of 0.82 had markedly unstable membranes compared to HS membranes with comparable spectrin density (27). These results indicate that the membrane instability of HE and HPP membranes is due to the combined influences of qualitative and quantitative deficiencies of spectrin, whereas that of HS membranes is a direct reflection of quantitative deficiencies in spectrin.

\section{Discussion}

The data presented here show that in spectrin-deficient $\mathrm{HS}$, the membrane mechanical stability is reduced, and that the extent of this reduction can be correlated with the decrease in membrane spectrin levels. Moreover, our finding that the reduction in membrane mechanical stability can be related to surface area loss implies that spectrin-deficiency-induced mechanical

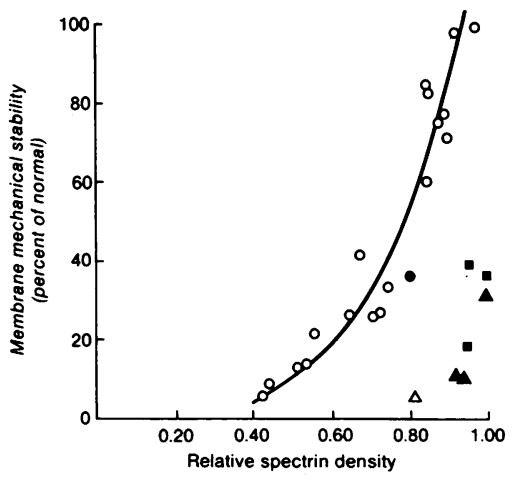

Figure 7. Membrane mechanical stability and spectrin density in various spectrin-deficient hemolytic anemias. Mildly spectrindeficient membranes from individuals with HPP $(\Delta)$, HE with a mutant $\alpha$ spectrin ( $\Delta$ ), and $\mathrm{HE}$ with abnormal $\alpha$ I domain tryptic peptides ( $\square$ ) have more marked membrane in-

stability than spectrin-deficient HS membranes (0) with similar spectrin content, implying that in these instances, additional molecular lesions are responsible for impaired membrane stability. However, HS with abnormal protein 4.1-spectrin association (•) resembles spectrin-deficient HS in its relationship between membrane mechanical stability and spectrin content. The membrane mechanical stability $(M S)$ in spectrin-deficient $\mathrm{HS}$ is related to the spectrin density $(S D)$ by the equation $M S=0.01 \mathrm{e}^{4.81 S D}(r=0.954)$. 


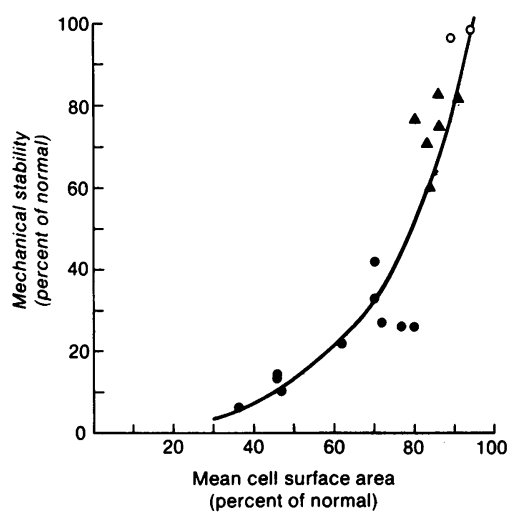

instability may be responsible for in vivo fragmentation, and cell surface area loss.

Having the measurements of both spectrin density and spectrin content enabled us to examine the relationships of these two parameters with surface area and membrane stability. Our results imply that whereas spectrin density more accurately reflects membrane surface area, spectrin content is a better indicator of alterations in membrane stability. The relationship between spectrin content and membrane mechanical properties in HS has also been suggested by recent micropipette studies in which membrane shear modulus was found to be related to spectrin content (23).

Our ability to directly assess cell surface area was important to this study. Using osmotic gradient ektacytometry, several erythrocyte parameters can be examined simultaneously. Not only does this technique measure surface area/volume ratio with a sensitivity similar to the standard osmotic fragility assay, but it also measures membrane surface area. Since the only other reliable measurement of erythrocyte membrane surface area is the more lengthy quantitation of total membrane lipids, ektacytometry may prove to be a sensitive and convenient alternative technique to study cell surface area loss in spherocytosis.

Spectrin deficiency also occurs in HPP and in HE with $\alpha$-spectrin variants. Interestingly, in these instances, the membranes are much more unstable than spectrin-deficient HS membranes with comparable spectrin density. Note that HE erythrocytes with unstable membranes have little loss of surface area, whereas HS erythrocytes with similarly unstable membranes have dramatic surface area loss. This difference may arise because of a basic difference in the underlying molecular lesions. HE and HPP involve molecular abnormalities in the regions of protein-protein associations, whereas HS involves a change in the density of the protein lattice underlying the lipid bilayer. From our data, it appears that the density of the spectrin lattice may be more important in maintaining surface area than intact protein-protein associations.

We have previously reported patients with spherocytic phenotype and little or no decrease in membrane mechanical stability (19). Three of the individuals described had erythrocyte membranes with reduced mechanical stability similar to that observed in the dominant HS patients in the current group. The remaining patients had mechanical stability values in the lower end of the normal range. Several factors may account for the normal membrane stability in these patients. The mechanical stability assay used in the present study has increased sensitivity to detect small reductions in mechanical stability that might previously have been missed. An additional or alternative explanation may be that since extensive clinical and biochemical data were not collected from the former group of patients, they may belong to the subgroup of HS with normal spectrin content and normal membrane stability (28).

Spectrin deficiency apparently does not play a role in all individuals with $\mathrm{HS}$. We have recently observed three patients with spherocytosis whose erythrocytes have sustained a surface area loss similar in degree to that seen in spectrin-deficient HS (28). However, compared with spectrin-deficient HS, these membranes contain normal membrane spectrin density and possess normal membrane stability. These individuals thus make up a separate subgroup of spherocytosis that occurs with relatively low frequency, and in whom the biochemical basis for the membrane loss is yet to be determined.

Although the biochemical basis for in vivo fragmentation in spectrin-deficient HS seems clear, the mechanism of the spectrin deficiency is unknown. We can speculate that studies using the recently isolated $\alpha$ - and $\beta$-spectrin gene probes will reveal a spectrum of abnormalities similar to those seen in the thalassemias. These could include defects in synthesis and stability of spectrin and defects in synthesis and stability of other membrane proteins essential for the assembly and maintenance of a normal membrane skeleton. In spectrin-deficient HS, the final amount of spectrin that is assembled on the membrane is important in determining the mechanical stability and thus the ability of the cells to maintain surface area during their life span.

\section{Acknowledgments}

We would like to thank Mr. James Harris for his excellent help in the preparation of this manuscript.

This work was supported in part by grants DK-26263, DK-32094, and HL-33991 from the U. S. Public Health Service. Dr. Agre is an Established Investigator of the American Heart Association.

\section{References}

1. Young, L. E. 1955. Observations on inheritance and heterogeneity of chronic spherocytosis. Trans. Assoc. Am. Phys. 68:141-148.

2. Dacie, J. V. 1960. The Haemolytic Anaemias: Congenital and Acquired. 2nd ed. Grune \& Stratton, Inc., New York. 82-150.

3. Agre, P., J. F. Casella, W. H. Zinkham, C. McMillan, and V. Bennett. 1985. Partial deficiency of erythrocyte spectrin in hereditary spherocytosis. Nature (Lond.). 314:380-383.

4. Agre, P., A. Asimos, J. F. Casella, and C. McMillan. 1986. Inheritance pattern and clinical response to splenectomy as a reflection of erythrocyte spectrin deficiency in hereditary spherocytosis. N. Engl. J. Med. 315:1579-1583.

5. Winkelman, J. C., S. L. Marchesi, P. Watkin, A. J. Linnenbach, P. Agre, and B. G. Forget. 1986. Recessive hereditary spherocytosis is associated with an abnormal alpha spectrin subunit. Clin. Res. 34:474A. (Abstr.)

6. Liu, S. C., L. H. Derick, P. Agre, and J. Palek. 1986. Alteration of the red cell membrane skeletal ultrastructure in hereditary spherocytosis. Blood. 68:56a. (Abstr.)

7. Goodman, S. R., K. A. Shiffer, L. A. Casoria, and M. E. Eyster. 
1982. Identification of the molecular defect in the erythrocyte membrane skeleton of some kindreds with hereditary spherocytosis. Blood. 60:772-784.

8. Wolfe, L. C., K. M. John, J. C. Falcone, A. M. Byrne, and S. E. Lux. 1982. A genetic defect in the binding of protein 4.1 to spectrin in a kindred with hereditary spherocytosis. N. Engl. J. Med. 307:13671373.

9. Becker, P. S., J. S. Morrow, and S. E. Lux. 1987. Abnormal oxidant sensitivity and $\beta$ chain structure of spectrin in hereditary spherocytosis associated with defective spectrin-4.1 binding. J. Clin. Invest. 80:557-565.

10. Coetzer, T. L., J. Lawler, S.-C. Liu, J. T. Prchal, R. J. Gualtieri, M. C. Brain, Sir J. V. Dacie, and J. Palek. 1988. Partial ankyrin and spectrin deficiency in severe, atypical hereditary spherocytosis. $N$. Engl. J. Med. 318:230-234.

11. Rybicki, A. C., R. Heath, J. L. Wolf, B. Lubin, and R. S. Schwartz. 1986. Deficiency of protein 4.2 in erythrocytes from a patient with Coombs negative hemolytic anemia. Blood. 68:57a. (Abstr.)

12. Fairbanks, G., T. L. Steck, and D. F. H. Wallach. 1971. Electrophoretic analysis of the major peptides of the human erythrocyte membrane. Biochemistry. 10:2606-2617.

13. Fenner, C., R. R. Traut, D. T. Mason, and J. Wikman-Coffelt. 1975. Quantification of Coomassie blue stained proteins in polyacrylamide gels based on analysis of eluted dye. Anal. Biochem. 63:595602.

14. Johnson, R. M. 1975. The kinetics of resealing washed erythrocyte ghosts. J. Membr. Biol. 22:231-253.

15. Heath, B. P., N. Mohandas, J. L. Wyatt, and S. B. Shohet. 1982. Deformability of isolated red blood cell membranes. Biochim. Biophys. Acta. 691:211-219.

16. Chasis, J. A., N. Mohandas, and S. B. Shohet. 1985. Erythrocyte membrane rigidity induced by glycophorin A-ligand interaction. Evidence for a ligand-induced association between glycophorin A and skeletal proteins. J. Clin. Invest. 75:1919-1926.

17. Evans, E. A., and A. Leung. 1984. Adhesivity and rigidity of erythrocyte membrane in relation to wheat germ agglutinin binding. $J$. Cell Biol. 98:1201-1208.
18. Mohandas, N., M. R. Clark, M. S. Jacobs, and S. B. Shohet. 1980. Analysis of factors regulating red cell deformability. J. Clin. Invest. 66:563-573.

19. Mohandas, N., M. R. Clark, B. P. Heath, M. Rossi, L. C. Wolfe, S. E. Lux, and S. B. Shohet. 1982. A technique to detect reduced mechanical stability of red cell membranes: relevance to elliptocytic disorders. Blood. 59:768-774.

20. Chasis, J. A., and N. Mohandas. 1986. Erythrocyte membrane deformability and stability: two distinct membrane properties that are independently regulated by skeletal protein associations. J. Cell Biol. 103:343-350.

21. Clark, M. R., N. Mohandas, and S. B. Shohet. 1983. Osmotic gradient ektacytometry: comprehensive characterization of red cell volume and surface maintenance. Blood. 61:899-910.

22. Mohandas, N., L. E. Lie-Injo, M. Friedman, and J. W. Mak. 1984. Rigid membranes of Malayan ovalocytes: a likely genetic barrier against malaria. Blood. 63:1385-1392.

23. Waugh, R. E. 1987. Effects of inherited membrane abnormalities on the viscoelastic properties of erythrocyte membrane. Biophys. $J$. 51:363-369.

24. Becker, P. S., and S. E. Lux. 1985. Hereditary spherocytosis and related disorders. Clin. Haematol. 14:15-43.

25. Lane, P., R. L. Shew, T. A. Iarocci, N. Mohandas, T. Hays, and W. C. Mentzer. 1987. Unique alpha-spectrin mutant in a kindred with common hereditary elliptocytosis. J. Clin. Invest. 79:989-996.

26. Mentzer, W. C., T. A. Iarocci, N. Mohandas, P. A. Lane, B. Smith, J. Lazerson, and T. Hays. 1987. Modulation of erythrocyte membrane mechanical stability by 2,3-diphosphoglycerate in the neonatal poikilocytosis/elliptocytosis syndrome. J. Clin. Invest. 79:942949.

27. Knowles, W. J., J. S. Morrow, D. W. Speicher, H. S. Zarkowsky, N. Mohandas, W. C. Mentzer, S. B. Shohet, and V. T. Marchesi. 1983. Molecular and functional changes in spectrin from patients with hereditary pyropoikilocytosis. J. Clin. Invest. 71:18671877.

28. Chasis, J. A., P. Agre, and N. Mohandas. 1987. The structural heterogeneity in congenital spherocytosis. Clin. Res. 35:422A. (Abstr.) 